\title{
Summary of Research Status and Application of MEMS Accelerometers
}

\author{
Weimeng Niu, Liqing Fang*, Lei Xu, Xu Li, Ruikun Huo, Deqing Guo, Ziyuan Qi \\ Army Engineering University Shijiazhuang Campus, Shijiazhuang, China \\ Email: *xldxulei@163.com
}

How to cite this paper: Niu, W.M., Fang, L.Q., Xu, L., Li, X., Huo, R.K., Guo, D.Q. and Qi, Z.Y. (2018) Summary of Research Status and Application of MEMS Accelerometers. Journal of Computer and Communications, 6, 215-221.

https://doi.org/10.4236/jcc.2018.612021

Received: December 19, 2018

Accepted: December 27, 2018

Published: December 30, 2018

\begin{abstract}
The rapid development of MEMS technology has made MEMS accelerometers mature and the application range has been expanded. Many kinds of MEMS accelerometers are researched. According to the working principle of MEMS accelerometer, it can be divided into: piezoresistive, piezoelectric, capacitive, tunnel, resonant, electromagnetic, thermocouple, optical, inductive, etc. Due to its outstanding features in terms of size, quality, power consumption and reliability, MEMS sensors are used in military applications and where high environmental resistance is required. MEMS accelerometers are developing rapidly and have good application prospects. In order to make MEMS accelerometers more widely understood, the advantages of MEMS accelerometers are expounded. The research status of MEMS accelerometers is introduced, and MEMS are analyzed. The application of accelerometers in real-world environments, and the development trend of MEMS accelerometers in the future. More scholars will invest in MEMS accelerometer research, pursuing high performance, low power consumption, high precision, multi-function, and interaction. Strong MEMS accelerometers will be ubiquitous in the future.
\end{abstract}

\section{Keywords}

MEMS Technology, MEMS Accelerometer

\section{Introduction}

The sensor based on MEMS technology is generally a highly integrated sensor system integrating micro-mechanical and micro-electronic functions, which is made of silicon-based materials and semiconductor integrated circuit manufacturing process. It has remarkable small size, light weight and low power consumption. Low cost, high reliability, strong resistance to vibration and shock, 
etc. At the same time, feature sizes on the order of micrometers allow them to perform functions that are not possible with some conventional sensors. The MEMS products currently being developed are mainly microelectromechanical sensors including MEMS gyroscopes, microelectromechanical accelerometers and micro pressure gauges [1]. Due to its outstanding features in terms of size, quality, power consumption and reliability, MEMS sensors are used in military applications and where high environmental resistance is required.

\section{Advantages of MEMS Accelerometers}

MEMS accelerometers are developing rapidly and have good application prospects. Their application advantages are mainly reflected in the following aspects:

1) The sensor is small in size and has high measurement accuracy. The micro accelerometer has experienced technical solutions such as interdigital, sandwich, and resonant beam. The zero bias and scale factor are $10 \mathrm{mg}$ from 2005 to $20 \mathrm{ug}$ in 2005. The micro-miniature two-axis accelerometer successfully developed by Analog Devices is only $5 \mathrm{~mm} \times 5 \mathrm{~mm} \times 1.45 \mathrm{~mm}$, with a mass of less than $1 \mathrm{~g}$ and a resolution of $10^{-3}$. The silicon microgyroscope ADXRS series measures 7 $\mathrm{mm} \times 7 \mathrm{~mm} \times 3 \mathrm{~mm}$ and has a mass of less than $1 \mathrm{~g}$. Continuous innovation of technical solutions reduces the source of error from the working mechanism and improves the accuracy.

2) High degree of integration, which can be mass-produced. Through the MEMS process, integration of multiple sensors with different functions and sensitive directions can be realized to form a micro-sensor array or micro-system. Similar to microelectronic chips, MEMS devices can be mass-produced and have low production costs, which is conducive to the industrialization of MEMS products.

3) Passivation can be achieved. In the Internet of Things era, networked measurement and control systems often require wireless MEMS sensors. As a sensor that converts non-electricity into electricity, power is a key point. The energy harvesting chip collects other energy sources such as solar energy and wind energy, and then converts it into electric energy to supply power to the sensor. The combination of the wireless sensing module and the energy harvesting technology will make the MEMS sensor passive.

\section{Research Status of MEMS Accelerometer}

According to the working principle of MEMS accelerometer, it can be divided into: piezoresistive, piezoelectric, capacitive, tunnel, resonant, electromagnetic, thermocouple, optical, inductive, etc. A typical MEMS accelerometer is introduced.

\subsection{Piezoresistive Acceleration Sensor}

The basic principle of the MEMS piezoresistive accelerometer is the piezoresistive effect. The inductive component is a varistor fabricated on a sensitive film or 
a sensitive beam. The sensing process is: when the object generates motion, the mass inside the accelerometer will under the action of the inertial force, the up and down motion is generated. Since the mass is supported by the cantilever beam, under the traction of the moving mass, the varistor located on the cantilever beam is deformed, and the resistance value changes, resulting in the power supply in Wheatstone. A small ripple voltage is generated in the bridge circuit, and the output signal of the Wheatstone bridge is amplified by the readout circuit, and the magnitude of the corresponding acceleration can be calculated by the calibration rule, and the change trend of the acceleration reflects the moving direction of the target [2] [3]. Figure 1 is a physical diagram of MEMS piezoresistive acceleration. For the MEMS piezoelectric acceleration sensor, only the varistor is replaced by a piezoelectric piece, and the piezoelectric effect is used to detect the acceleration change, and the working principle is similar to the piezoresistive type. The MEMS piezoresistive accelerometer is a micro-acceleration sensor that was studied earlier. It has formed a relatively mature design theory system in the course of many years of research, and it has been applied in practice in the 1980s. The main disadvantages of piezoelectric and piezoresistive accelerometers include the material's obvious fatigue effect, low sensitivity, large nonlinear error, and easy exposure to temperature. Future developments focus on improving sensitivity and reducing cross-coupling [4].

\subsection{Capacitive Accelerometer}

MEMS capacitive accelerometers use the change of capacitance to test the acceleration change, which is one of the most widely used methods in many measurement methods [5]. The sensor is generally composed of a sensitive structure and a fixing mechanism, and constitutes a variable capacitance dynamic capacitor. When the acceleration changes, the capacitance between the sensitive structure and the fixing mechanism also changes, and the peripheral detection circuit can the amount of change is tested and the value of the true acceleration of the object can be measured indirectly based on the acceleration calibration. MEMS capacitive accelerometers have the advantages of low energy consumption, good linearity, high measurement accuracy, stable test process, etc. At the same time, there are also defects such as complex signal processing circuits and poor anti-electromagnetic interference capability. COMS integration, single-chip multi-axis and other directions. The capacitive accelerometer is shown in Figure 2.

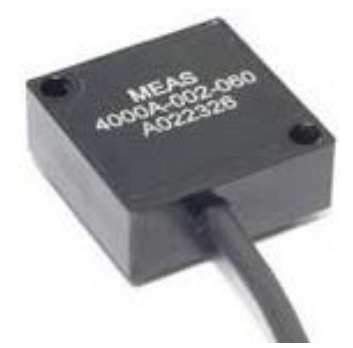

Figure 1. Piezoresistive accelerometer. 


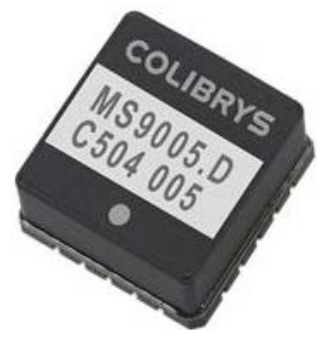

Figure 2. Capacitive accelerometer.

\subsection{Tunnel Accelerometer}

Using tunneling to study the displacement and tunneling current is the basic principle of MEMS tunneling accelerometer [6]. At room temperature, when the distance between the two electrodes is very close, the voltage of the electric field is continuously enhanced by the excitation of the geometric shape and the like. When the reduction is small enough, the electrons between the metal electrodes will actively penetrate. Generate tunnel current. When subjected to inertial force, the sensitive block is displaced, and the distance between the electrodes changes. By measuring the value of the current, the magnitude of the external acceleration can be calculated. These sensors are characterized by strong anti-interference ability, high reliability, easy detection, and small temperature effect. However, since quantum effect is the basis of such sensors, the theoretical model is relatively immature and based on the volume and size of MEMS tunnel-type accelerometers. The limitation is greatly affected by noise and the manufacturing process is also complicated.

\subsection{Resonant Accelerometer}

The MEMS resonant accelerometer uses the frequency signal to achieve the purpose of measurement [7]. The resonant beam is the core component of such an accelerometer. When the object has an acceleration output, the inertial force drives the mass to vibrate, and the resonant beam is in the mass. The deformation occurs under the influence of the natural frequency. By detecting the resonance frequency in this process, the excitation amount is calculated and the magnitude of the acceleration is obtained. The MEMS resonant accelerometer can detect the frequency signal of the moving object and use the characteristics of the remaining input acceleration to achieve the purpose of measuring the acceleration. Since the frequency signal approximates the digital signal, it can be directly accepted by the digital processing chip, so it has a good resolution. And the sensitivity, the detection circuit is very simplified, and the stability is high in the process of signal transmission. However, since it takes a long time to integrate its high-resolution signal, realizing its rapid real-time detection is the development direction of such sensors.

With the continuous development of science and technology, the types of MEMS accelerometers are continuously enriched, and the limitations are becoming smaller and smaller. Devices developed with new principles are con- 
stantly emerging. The thermal convection acceleration sensor designed by temperature difference has good structural stability and strong impact resistance. The accelerometer based on acousto-optic effect breaks through the compatible processing technology of single crystal quartz body processing and patterning; using magnetic fluid to design acceleration sensor to overcome the common sensor contact points wear each other. In addition, there are fiber-optic, laser self-mixing effect, thermocouple type, the new principle is more prominent in the new sensor, and the application in weapons and equipment will be more extensive.

\section{MEMS Accelerometer Application Status}

MEMS accelerometers are widely used in many fields. The wearable data device SCUPPY developed by Samsung uses gyroscopes to sense the direction of motion, and MEMS accelerometers sense the motion generated by finger movements; Cao Yuzhen et al. [8] The human body falls or not, and the human body attitude detection technology is developed with the MEMS accelerometer as the core. The acceleration vector magnitude and the differential value of the vector amplitude are used to sense the movement of the human body. In the field of human-computer interaction, Nintendo launched the WII game controller. MEMS accelerometer is used to detect and identify any space motion [9] [10]; Su Weijia [11] and other integrated accelerometers and microprocessors design an automotive brake detection system that can intelligently and conveniently detect the brakes of the car. Stability and braking efficiency; the small size of the MEMS accelerometer makes it suitable for portable ultra-small-sized consumer products such as mobile phones and notebook computers [12]; MEMS accelerometers have been moving toward high precision and high integration. It has been able to meet equipment guidance and control in terms of accuracy required [13]. The PIMPF (Programmable Intelligent Multi-Purpose Fuze) fuze developed by Germany and France and the MEHTF (Multiple-Event Hart Target Fuze) developed by the United States on the basis of HTSF (Hart Target Smart Fuze) are two representative intelligent penetration fuzes. High G-value accelerometer for acceleration signal recognition and signal acquisition, data processing and burst control using a microcontroller [14]; France uses a MEMS accelerometer to design a smart fuze that can withstand an impact of approximately 100,000 G. The fuze can send the necessary information such as the reached depth and the number of layers penetrated to the projectile control system at the moment of the detonation of the projectile, and perform intelligent analysis processing in the background. According to the relevant information of the process, the fuze can be obtained. Whether the enemy's command center was destroyed when the explosion occurred, and whether the enemy's command center was destroyed [15]; the MEMS fuze safety insurance mechanism developed by the US Navy using MEMS accelerometers has the characteristics of reliability and high adaptability to the environment [16]. Inside the fuze, the magnitude of the torpedo acceleration is transmitted by acceleration The sensor senses, the first insurance 
is released when the predetermined value is reached, and the output signal is transmitted to the next actuator, which fully displays the intelligent characteristics of the ammunition; the acceleration sensor acts as the MIMU (Micro Inertial Measurement Unit). The performance of the core device directly determines the performance of the system. The MIMU is used to detect the attitude of the projectile during flight. By combining with GPS, the flight path of the projectile can be accurately detected. The combined system can accurately control the projectile. Detonate the point and correct the ballistics [17].

\section{Outlook}

With the development of micro-engineering and electronic system integrated integration design, using FPGA, ASIC, ASIS, SOC, IP, MEMS and other advanced technologies to achieve system-level miniaturization and integrated design, it will be a design style of military aircraft in the 21 st century. MEMS technology is developing rapidly and has outstanding features. Various types of accelerometers based on MEMS have been widely used in various fields such as industry, aviation, military, communications, etc. With the continuous advancement of science and technology, knowledge and technology of various disciplines are continuously integrated, and scholars of different research directions are from different sides. Focus on improving and innovating MEMS accelerometers. In the era of intelligent Internet of Things, MEMS accelerometers are welcoming opportunities and facing challenges. More scholars will invest in MEMS accelerometer research, pursuing high performance, low power consumption, high precision, multi-function, and interaction. Strong MEMS accelerometers will be ubiquitous in the future.

\section{Conflicts of Interest}

The authors declare no conflicts of interest regarding the publication of this paper.

\section{References}

[1] Amarasinghe, R., Dao, D.V., Toriyama, T., et al. (2007) Development of Miniaturized 6-Axis Accelerometer Utilizing Piezoresistive Sensing Elements. Sensors and Actuators A: Phsical, 134, 310-320. https://doi.org/10.1016/j.sna.2006.05.044

[2] Tsai, M.H., Liu, Y.C., Sun, C.M., et al. (2010) A CMOS-MEMS Accelerometer with Tri-Axis Sensing Electrodes Arrays. Procedia Engineering, 5, 1083-1086. https://doi.org/10.1016/j.proeng.2010.09.298

[3] Kanda, K., Iga, Y., Matsuoka, J., et al. (2010) A Tri-Axial Accelerometer with Structure-Based Voltage Operation by Using Series-Connected Piezoelectric Elements. Procedia Engineering, 5, 894-897. https://doi.org/10.1016/j.proeng.2010.09.253

[4] Yoon, K., Soh, S., Seok, B., et al. (2005) A New Wearable Input Device: SCURRY. IEEE Transaction on Industrial Electronics, 52, 1490-1499. https://doi.org/10.1109/TIE.2005.858736

[5] Rockstad, H.K., Tang, T.K., Reynolds, J.K., Henny, T.W., Kaiser, W.J. and Gabrielson, T.B. (1996) A Miniature High-Sensitivity Electron Tunneling Accelerometer. 
Sensors and Actuators A, 53, 227-231. https://doi.org/10.1016/0924-4247(96)01128-4

[6] Aaltonen, L. and Halonen, K. (2009) Continuous-Time Interface for a Micromachined Capacitive Accelerometer with NEA of $4 \mu \mathrm{g}$ and Bandwidth of $300 \mathrm{~Hz}$. Sensors and Actuators A: Phsical, 154, 6-56.

[7] Pinto, D., Mercier, D., Kharrat, C., et al. (2009) A Mall and High Sensitivity Resonant Accelerometer. Procedia Chemistry, 1, 536-539. https://doi.org/10.1016/j.proche.2009.07.134

[8] Cao, Y.Z., Cai, W.C. and Cheng, W. (2010) Human Body Attitude Detection Technology Based on MEMS Accelerometer. Nanotechnology and Precision Engineering, No. 1, 37-40.

[9] Thomas, S., Benjamin, P., Niels, H., et al. (2008) Gesture Recognition with A Wii Controller. Second International Conference on Tangible and Embedded Interaction-Conference Proceedings, No. 16, 11-14.

[10] Chung, L.J. (2008) Hacking the Nintendo Wii Remote. IEEE Pervasive Computing, 7, 39-45. https://doi.org/10.1109/MPRV.2008.53

[11] Su, W.J. and Su, J. (2008) Application of Acceleration Sensor in Vehicle Braking Performance Detection. Industrial Control Computer, No. 2, 74-75.

[12] Kevin, T.C.C., Han, D., Ravinder, P.S., et al. (2010) 118-dB Namic Range, Continuous-Time, Opened-Loop Capactance to Voltage Converter Readout for Capacitive MEMS Accelerometer. Solid State Circuits Conference. IEEE, 1-4.

[13] Chen, W. (2014) Research on Key Technology of High Precision Attitude Measurement Based on MEMS Inertial Sensor. Master's Thesis, Zhejiang University, Hangzhou.

[14] Brown, T. and Davis, B. (2001) Strap-Down Micro-Electro-Mechanical (MEMS) Sensors for Higher Munition Applications. IEEE Transactions on Magnetic, 37, 336-342. https://doi.org/10.1109/20.911850

[15] Dang, A.G. and Li, X.J. (2014) Review and Prospect of the Development of Earth-Drilling Weapons Abroad. No. 6, 35-39.

[16] Last, H., Deeds, M., Garvic, D., et al. (1999) Nanoto Milli-Meter Scale Integrated Systems. IEEE/Tansactions on Components and Packaging Technologies, No. 22, 138-142.

[17] Shen, Q. and Li, S.Y. (2002) Calculation of Ballistic Parameters of Ballistic Correction Fuze Based on MIMU and Its Precision Requirements for MIMU. Journal of Detection and Control, 24, 35-39. 